PROCEEDINGS OF THE

AMERICAN MATHEMATICAL SOCIETY

Volume 126, Number 12, December 1998, Pages 3447-3454

S 0002-9939(98)05000-X

\title{
SPLITTING OF THE DIRECT IMAGE OF SHEAVES UNDER THE FROBENIUS
}

\author{
RIKARD BØGVAD
}

(Communicated by Ron Donagi)

\begin{abstract}
A generalisation and a new proof are given of a recent result of J. F. Thomsen (1996), which says that for $L$ a line bundle on a smooth toric variety $X$ over a field of positive characteristic, the direct image $F_{*} L$ under the Frobenius morphism splits into a direct sum of line bundles. (The special case of projective space is due to Hartshorne.) Our method is to interpret the result in terms of Grothendieck differential operators $\operatorname{Diff}^{(1)}(L, L) \cong$ $\operatorname{Hom}_{O_{X}(1)}\left(F_{*} L, F_{*} L\right)$, and $T$-linearized sheaves.
\end{abstract}

\section{INTRODUCTION}

All varieties are, for simplicity, assumed to be defined over an algebraically closed field $k$ of positive characteristic $p$.

Our argument for the theorem of Thomsen, described in the abstract, is as follows: On a toric variety all invertible sheaves $L$ are equipped with a linearization over the torus $T([\mathrm{~F}, 3.4])$. This implies naturally that the algebra of distributions on $T$, Dist ${ }^{(1)}(T)$, acts on $F_{*} L$, and hence this module splits as a direct sum of locally free modules according to the central idempotents. By restricting to the open orbit, and using that $\operatorname{Dist}^{(1)}(T)$ is commutative semi-simple, one sees then that the summands have rank 1 . This argument amounts to using global differential operators, and it enables us to avoid the description of the toric variety in terms of a fan, which Thomsen uses, though it is, of course, really only a slightly fancy way to work with gradings on $L$. Furthermore the argument, applied to an arbitrary, not necessarily locally free, $T$-linearized module $M$, on a not necessarily smooth toric variety, continues to have some content. It then says that $F_{*} M$ splits into a sum of $p^{n} T$-linearized modules, where the rank of $T$ is $n$ (Theorem 2). We will now explain the terminology and give details.

\section{LinEARIZATION}

Suppose that $G$ is an affine algebraic group and $X$ an algebraic variety with an action $\mu: G \times X \rightarrow X$ of $G$. Let $p: G \times X \rightarrow X$ be the projection on the second factor. Then a quasi-coherent $O_{X}$-module $M$ is $G$-linearized, if there is an isomorphism $\theta: \mu^{*} M \cong p^{*} M$, satisfying a certain relation, which may be found in

Received by the editors November 1, 1996.

1991 Mathematics Subject Classification. Primary 14M25; Secondary 14F05, 14L17.

(C)1998 American Mathematical Society 
e.g. Mumford's book on geometric invariant theory [M, I.3, p. 30]. If $X$ is affine, $\theta$ defines a map

$$
\theta_{c}: M \rightarrow \mu^{*} M \rightarrow p^{*} M \cong O_{G} \otimes_{k} M,
$$

and the relation then says that $M$ is a comodule for the Hopf algebra $O_{G}$. Dually, if $g \in G(k)$ defines a map $g: X \ni x \mapsto g x \in X$, then by pulling back to a section $g \times X \subset G \times X$, the existence of $\theta$ implies that there is an isomorphism $g^{*} M \cong M$, and the relation then says that these isomorphisms compose compatibly with group multiplication.

On toric varieties vector bundles, linearized with respect to the torus, have been studied by Kaneyama [K1], [K2], while linearized modules that are not even necessarily coherent have been used in $D$-module-theory on Borel varieties (see e.g. [B1, VII.12.11], [B]). Interesting examples are local cohomology modules $H_{Y}^{i}\left(X, O_{X}\right)$, where $Y$ is an orbit for $G$. Kaneyama gives an equivalent description of linearized vector bundles in terms of $T$-graded modules associated with the fan, which is possible to adapt to general $T$-linearized sheaves.

In general linearized modules are, of course, nicest on orbits. Suppose that $\pi: G \rightarrow X=G / H$, for a subgroup $H \in G$. Pulling back a $G$-linearized coherent $O_{X}$-module $M$ by $\pi$ gives a $G$-linearized coherent $O_{G}$-module, and hence it follows, by faithful flatness of $\pi$ and the first part of the following proposition, that $M$ is locally free (in fact it is possible to see that $G$-linearized modules are precisely sheaves induced from $H$-modules, in the sense of e.g. [J, I.5.8]).

Proposition 2.1. The quasi-coherent $O_{G}$-module $M$ is $G$-linearized if and only if it is a bimodule for the Hopf algebra $O_{G}$. In particular $M \cong O_{G} \otimes_{k} V$, for some vector space $V$, as bimodules, and hence is locally free. A quasi-coherent $G$-linearized module on $G / H$ for $H$ a closed subgroup is locally free.

Proof. The second statement follows from Theorem 3.1.8 in [A]. The first is an easy verification, while the proof of the last statement was given above.

\section{Distributions as Differential operators ON DIRECT IMAGES OF FrobENIUS}

Let $I$ be the ideal of the identity $1 \in G$ and $\left(I^{\left(p^{r}\right)}\right)$ the ideal in $O_{G}$ which is generated by $p^{r}$-th power of elements in $I$. Define the algebra $\operatorname{Dist}^{(r)}(G)$ of distributions of order less than $p^{r}$ as $\operatorname{Hom}\left(O_{G} /\left(I^{\left(p^{r}\right)}\right), k\right)$, with an algebra structure induced by the comultiplication $O_{G} \rightarrow O_{G} \otimes_{k} O_{G}$. (Cf. e.g. [J, Chap. 7].) It may also be identified with the left invariant global differential operators corresponding to left multiplication of $G$ on $G$ [J, I, 7.18]. Considered as such

$$
p(g):=\left(p \otimes 1_{O_{G}}\right)(\Delta(g)),
$$

for $g \in O_{G}, p \in \operatorname{Dist}^{(r)}(G)$, and where $\Delta$ is the coproduct of $O_{G}$. Note that naturally $\operatorname{Dist}^{(r)}(G) \subset \operatorname{Dist}^{(r+1)}(G)$, and let $\operatorname{Dist}(G)$ be the direct limit.

This definition is intimately connected with the Frobenius $F:=F_{X / k}$, defined by the diagram

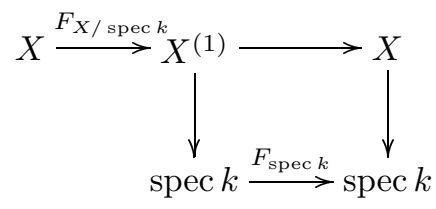


where all horizontal maps are the identity on the topological spaces; the lower horizontal map is just the $p$-th power map on $k$, the square is a pullback diagram, and the composition of the upper horizontal maps is the $p$-th power map. Note that the assumption that $k$ is algebraically closed implies that $X \cong X^{(1)}$, though not as $k$-varieties. The following proposition says that the algebra of distributions acts as differential operators on any linearized module.

Proposition 3.1. If the quasi-coherent $O_{X}$-module $M$ is $G$-linearized, then $\operatorname{Dist}^{(r)}(G)$ acts as a $k$-algebra on $F_{*}^{r} M$; in fact there is a natural algebra homomorphism

$$
\operatorname{Dist}^{(r)}(G) \rightarrow \operatorname{Diff}^{(r)}(M, M) \cong \operatorname{Hom}_{O_{X}(r)}\left(F_{*}^{r} M, F_{*}^{r} M\right) .
$$

Proof. (The proof is, for readability, formulated as if $X$ was affine, that is, suppressing all topological information.) From the linearization there is obtained a map

$$
\theta: M \rightarrow \mu^{*} M \rightarrow p^{*} M \cong O_{G} \otimes_{k} M
$$

and by definition of $\operatorname{Dist}^{(r)}(G)$ there is a map $m$ : $\operatorname{Dist}^{(r)}(G) \otimes_{k} O_{G} \rightarrow k$. The action of $u \in \operatorname{Dist}^{(r)}(G)$ is then defined as

$$
\operatorname{Dist}^{(r)}(G) \otimes_{k} M \stackrel{1 \otimes \theta}{\longrightarrow} \operatorname{Dist}^{(r)}(G) \otimes_{k} O_{G} \otimes_{k} M \stackrel{m \otimes 1_{M}}{\longrightarrow} M .
$$

(Note that this also gives the action of $\operatorname{Dist}^{(r)}(G)$ as differential operators on $O_{G}$.) The map above actually commutes with multiplication by $f \in O_{X^{(r)}}$ : Note first that, corresponding to the statement that 1 acts as the identity on $X$, the composite map

$$
O_{X} \stackrel{\mu^{*}}{\rightarrow} O_{G} \otimes_{k} O_{X} \stackrel{\alpha \otimes \mathrm{Id}}{\longrightarrow}\left(O_{G} / I\right) \otimes_{k} O_{X} \cong O_{X}
$$

where $\alpha$ is the map corresponding to the inclusion $1 \in G$, is the identity. This means that, if $f \in O_{X}$ and $\mu^{*}(f)=\sum g_{i} \otimes f_{i}$, then $f=\sum \alpha\left(g_{i}\right) f_{i}$. Secondly, if $h \in O_{G^{(r)}}$, then $h \sim \alpha(h)\left(\bmod I^{(r)}\right)$ and hence $p(h k)=\alpha(h) p(k)$, if $k \in O_{G}$ and $p \in \operatorname{Dist}^{(r)}(G)$. Assume now that $\theta(m)=\sum h_{i} \otimes m_{i}$, that $f \in O_{X^{(r)}}$ and $\mu^{*}(f)=\sum g_{i} \otimes f_{i}$ and note that $f_{i} \in O_{X^{(r)}}$ and $g_{i} \in O_{G^{(r)}}$. Then

$$
\begin{aligned}
p(f m) & =p\left(\mu^{*}(f)(\theta m)\right)=\sum_{i j} p\left(g_{j} h_{i}\right) \otimes f_{j} m_{i}=\sum_{i j} \alpha\left(g_{j}\right) p\left(h_{i}\right) \otimes f_{j} m_{i} \\
& =\sum_{i} p\left(h_{i}\right) \otimes\left(\sum_{j} \alpha\left(g_{j}\right) f_{j}\right) m_{i}=f(p m)
\end{aligned}
$$

as was to be shown. We omit the verification that the map is an algebra homomorphism. The identification with differential operators, due to Cartier, may be found in e.g. $[\mathrm{B}, \mathrm{Y}]$ and in the references given there.

\section{Distributions ON TORII}

The preceding proposition thus implies that the direct image under the Frobenius of a $G$-linearized $O_{X}$-module splits into a direct sum of $O_{X}$-modules according to the central idempotents in $\operatorname{Dist}^{(r)}(G)$. When are there many such? By the result of Nagata, formulated for group schemes in [DG, IV.3.3.6, p. 509] the only connected affine algebraic groups (in positive characteristics) which are linearly reduced - all representations split into a direct sum of simple representations - are 
the multiplicative groups. By the identification of Dist ${ }^{(r)}(G)$-modules and modules over the infinitesimal Frobenius kernels $G_{(r)}$ (see [J, I.8.6, 9.4]), this implies that the torii (together with infinitesimal subgroups) are the only connected affine algebraic group schemes with algebras of distributions that are semi-simple as rings. So now let the group be a torus $T$, of rank $n$ and identify

$$
O_{T} \cong k\left[x_{1}, x_{1}^{-1}, \ldots, x_{n}, x_{n}^{-1}\right] .
$$

Then $\operatorname{Dist}^{(r)}(T) \cong \prod_{i=1}^{i=n} \operatorname{Dist}^{(r)}\left(G_{m}\right)$ is described in e.g. [J], and it is indeed easy to construct an algebra isomorphism

$$
\operatorname{Dist}^{(r)}(T) \cong \bigoplus_{i=1}^{i=\left(p^{r}-1\right) n} k
$$

by exhibiting the corresponding central idempotents explicitly. We do this for completeness. Let $X^{(r)}(T)$ be the set of characters $\lambda$ of $T$ of the form $\left(t_{1} \cdots t_{n}\right) \rightarrow$ $t_{1}^{\lambda_{1}} \cdots t_{n}^{\lambda_{n}}$, where $\lambda=\left(\lambda_{1}, \ldots, \lambda_{n}\right)$ and $0 \leq \lambda_{s}<p^{r}$, for $1 \leq s \leq n$. The idempotents may be calculated as follows. If $m$ is a positive integer, let $m=\sum_{i \geq 0} m^{(i)} p^{i}$, $0 \leq m^{(i)} \leq p-1$, be its $p$-adical expression. Then for $\lambda \in X^{(r)}(T)$, define

$$
P_{\lambda}=\prod_{s=1}^{s=n} \prod_{j=1}^{j=r-1} \prod_{\substack{i=0 \\ i \neq \lambda_{s}^{(j)}}}^{i=p-1}(-1)^{\lambda_{s}^{(j)}-1}\left(i-\delta_{s}^{\left(p^{j}\right)}\right) .
$$

Here $\delta_{s}^{\left(p^{j}\right)}$ denotes the differential operator acting on $k\left[T_{s}, T_{s}^{-1}\right]$ by $\delta_{s}^{\left(p^{j}\right)} T_{s}^{k}=\left(\begin{array}{c}k \\ p^{j}\end{array}\right)=$ $\left(\begin{array}{c}k^{(j)} \\ 1\end{array}\right)=k^{(j)}[\mathrm{J}, 7.8]$. (We use the fact that, $\bmod p,\left(\begin{array}{c}m \\ k\end{array}\right)=\prod_{i}\left(\begin{array}{c}m_{i} \\ k_{i}\end{array}\right)$.) An application of Wilson's theorem gives that $P_{\lambda} T^{k}=T^{k}$ if $k=\lambda\left(\bmod p^{r}\right)$ and $P_{\lambda} T^{k}=0$ otherwise, and hence that the $P_{\lambda}$ are indeed idempotent, acting on the faithful module $O_{T}$. That they form a complete system follows from the fact that given a $\operatorname{Dist}(T)$-module $M \cong \bigoplus_{\mu} M_{\mu}$, where the $\mu$ are weights,

$$
P_{\lambda} M=\bigoplus_{\mu=\lambda} M_{\mu}
$$

\section{The Splitting of Direct images under the Frobenius ON TORIC VARIETIES}

Theorem 1 (compare $[\mathrm{T}]$ ). Let $X$ be a smooth toric variety and let $M$ be a $T$ linearized quasi-coherent $O_{X}$-module. For any integer $r \geq 1$

$$
F_{*}^{r} M \cong \bigoplus_{\lambda \in X^{r}(T)} P_{\lambda} M
$$

If $M$ is locally free, then each $P_{\lambda} M$ is also locally free, and the rank of $P_{\lambda} M$ is equal to the rank of $M$. In particular, for $L$ an invertible sheaf $F_{*}^{r} L$ is isomorphic to a direct sum of invertible sheaves.

Proof. If $X$ is smooth, then $F_{*}^{r} O_{X}$ is locally free and if furthermore $M$ is locally free, then $F_{*}^{r} M$ is also locally free. As a direct summand of a locally free module, it is then clear that $P_{\lambda} M$ is locally free. To check the equality of ranks, it suffices 
to restrict to the open orbit $T$ and consider $O_{T}$, by Proposition 2.1, and then the assertion is immediate, since

$$
P_{\lambda} O_{T}=\bigoplus_{\alpha \sim \lambda} k x^{\alpha} \cong O_{T^{(r)}} x^{\lambda} .
$$

For a toric variety, a line bundle is $T$-linearizable [F, 3.4], and the result of Thomsen follows.

The following is an extension of this result.

Theorem 2. Let $X$ be a toric variety and let $M$ be a T-linearized quasi-coherent $O_{X}$-module. For any integer $r \geq 1$

$$
F_{*}^{r} M \cong \bigoplus_{\lambda \in X^{r}(T)} P_{\lambda} M
$$

where the $P_{\lambda} M$ are $T^{(r)} \cong T$-linearized quasi-coherent modules on $X^{(r)} \cong X$.

Proof. Set $P:=P_{\lambda}$. The following example perhaps makes the proof more enlightening. Take $M=O_{T}$ itself; it is an $O_{T}$-comodule with $\theta\left(T^{k}\right):=\Delta T^{k}=T^{k} \otimes T^{k}$. Note first that $P O_{T}=\bigoplus_{\alpha \sim \lambda(\bmod p)} k x^{\alpha} \cong O_{T^{(r)}} x^{\lambda} \stackrel{\eta}{\cong} O_{T^{(r)}}$ is a subcoalgebra, isomorphic as a coalgebra to the Hopf algebra $O_{T^{(r)}}$. (That $P O_{G}$ is a subcoalgebra is easily seen to be true for any group $G$ and central idempotent $P \in \operatorname{Dist}^{(r)}(G)$.) Now define the $T^{(r)}$-linearization by $P_{\theta}: P O_{T} \rightarrow O_{T^{(r)}} \otimes P O_{T}, P_{\theta}=(\eta \otimes 1) \circ \theta$, or $P_{\theta}\left(T^{\lambda+p \beta}\right)=T^{p \beta} \otimes T^{\lambda+p \beta}$. This has the desired properties, since, with this

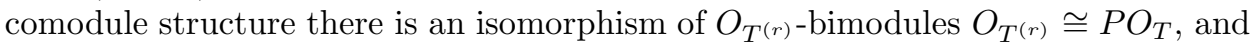
$O_{T^{(r)}}$ certainly is linearized (that the $O_{G}$-module $M$ is a bimodule means that the map $\theta: M \rightarrow O_{G} \times M$ satisfies $\theta(f m)=\Delta(f) \theta m$, and this clearly is what is needed for the comodule map $\theta$ to induce a linearization $\left.\Delta^{*} M \rightarrow p^{*} M\right)$.

Now first suppose that $X$ is affine and that $\theta: M \rightarrow O_{G} \otimes M$ is the comodule map. We first want to show that

$$
\theta: P M \rightarrow P O_{T} \otimes P M .
$$

Since the hyper-algebra is cocommutative (or $P$-central), $\left(P \otimes 1_{O_{G}}\right) g=\left(1_{O_{G}} \otimes P\right) g$, $g \in O_{G}$, and since $P$ is an idempotent, $(P \otimes P) \circ \Delta=P$. Hence, remembering that $\Delta \otimes 1_{M} \circ \theta=1_{O_{T}} \otimes \theta \circ \theta$, we have

$$
\begin{aligned}
\left(1_{O_{G}} \otimes P\right)(\theta m) & =\left(1_{O_{G}} \otimes P \otimes 1_{M}\right)\left(\left(1_{O_{G}} \otimes \theta\right)(\theta m)\right) \\
& =\left(1_{O_{G}} \otimes P \otimes 1_{M}\right)\left(\left(\Delta \otimes 1_{M}\right)\left(\theta_{m}\right)\right) \\
& =\left(P \otimes 1_{O_{G}} \otimes 1_{M}\right)\left(\left(\Delta \otimes 1_{M}\right)(\theta m)\right) \\
& =\left(P \otimes 1_{O_{G}} \otimes 1_{M}\right)\left(\left(1_{O_{G}} \otimes \theta\right)\left(\theta_{m}\right)\right)=(P \otimes \theta)(\theta m) \\
& =\theta\left(\left(P \times 1_{M}\right)(\theta m)\right)=\theta(P m),
\end{aligned}
$$

and similarly

$$
\begin{aligned}
\left(P \otimes 1_{M}\right)(\theta m) & =\left(P \otimes 1_{O_{G}} \otimes 1_{M}\right)\left(\left(\Delta \times 1_{M}\right)(\theta m)\right) \\
& =\left(P \otimes 1_{O_{G}} \otimes 1_{M}\right)\left(\left(1_{O_{G}} \otimes \theta\right)(\theta m)\right)=(P \otimes \theta)(\theta m) \\
& =\theta\left(\left(P \times 1_{M}\right) \theta m\right)=\theta(P m) .
\end{aligned}
$$

The counit $p O_{G} \rightarrow k$ is defined by restriction. Hence we have shown that $P M$ is a comodule for $P O_{T}$. Now, precisely as in the example of $O_{T}$ above, use the coalgebra isomorphism $P O_{T} \cong O_{T^{(r)}}$, to define an $(\eta \otimes 1)$-comodule structure 
$\theta_{\lambda}=(\eta \otimes 1) \circ \theta: P M \rightarrow O_{T^{(r)}} \otimes P M$. Let us first check that this structure is compatible with multiplication by elements $f \in O_{X^{(r)}}$, and hence such that $\theta f \in O_{G^{(r)}} \otimes O_{X^{(r)}}$ (we use $\theta$ for the comodule map of $O_{X}$, which by restriction gives the comodule map of $\left.O_{X^{(r)}}\right)$. This means that $\theta_{\lambda}(f m)=(\theta f) \theta_{\lambda} m$, if $m \in P M$. But this follows from the corresponding property of $M$, combined with the fact that $P$ commutes with all $p$-th powers, which implies that $\eta$ is $O_{T^{(r)}}$-linear.

It only remains to check that the induced linearization map is an isomorphism. We need more information on the comodules for $O_{T}$. Namely that representations split into homogeneous components, and that if $\theta m=\sum_{i} g_{i} \otimes m_{i}$, then $g_{i}^{-1} \in O_{T}$, but this is clear, since, if $m=\sum_{\mu \in X(T)} m_{\mu}$ is a decomposition into homogeneous elements, then $\theta m=\sum_{\mu \in X(T)} T^{\mu} \otimes m_{\mu}$ ( $T^{\mu}$ is the monomial with weight $\mu$ ). Consider then the commutative diagram

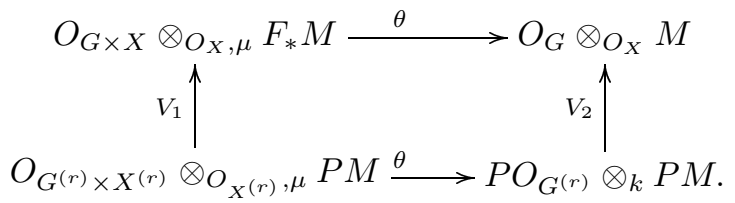

The vertical maps are the obvious ones based on inclusions of the modules concerned. There is an evident splitting of $V_{2}$ defined by $\Pi_{2}(g \otimes m)=P(g) \otimes P(m)$ and correspondingly a splitting of $V_{1}$ is defined by

$$
\Pi_{1}(g \otimes m):=P_{\lambda-\nu(m)}(g) \otimes P(m)
$$

if $g \in O_{T}, m \in M_{\nu(m)}$. Here $P_{\lambda-\nu(m)}$ is the projection operator in $\operatorname{Dist}^{(r)}(T)$ belonging to $\lambda-\nu(m) \in X^{(r)}(T)=X(T) / p^{r} X(T)$. This is a well-defined map, noting first that the relation $g x \otimes m=g T^{-\nu(x)} \otimes x m$, if $x \in\left(O_{X}\right)_{\nu(x)}$, means that every element in the definition set may be written in the form $g \otimes m$. Secondly, $P(m) \neq 0$ implies that $\lambda=\nu(m)\left(\bmod p^{r}\right)$, and hence $P_{\lambda-\nu(m)}(g) \in O_{T^{(r)}}$, so that the map goes to the right target also.

It is also easy to check that $\Pi_{1} \circ V_{1}=1$. Assume everything homogeneous: $\Pi_{1}(g \otimes m):=P_{\lambda-\nu(m)}(g) \otimes P(m)=g \otimes m$, if $m \in P(M)$, implying that $\nu(m) \sim \lambda$ $\left(\bmod p^{r}\right)$, and if $g \in O_{T^{(r)}}$. This implies that $V_{1}$ is an injection and hence the lower $\theta$ is also an injection, by commutativity of the diagram. Since moreover $\Pi_{2} \circ \theta=\theta \circ \Pi_{1}$, since $\Pi_{2} \circ \theta(g \otimes m)=P\left(g T^{\nu(m)}\right) \otimes P(m)$, while $\theta \circ \Pi_{1}(g \otimes m)=$ $\theta\left(P_{\lambda-\nu(m)}(g) \otimes P(m)\right)=\left(P_{\lambda-\nu(m)}(g) T^{\nu(m)}\right) \otimes P(m)$, it is clear that $\theta$ is a surjection.

The definition of the linearization clearly glues from $T$-invariant open subvarieties and hence this finishes the proof of the theorem.

(It should perhaps be noted that it is possible to give a version for actions of infinitesimal groups as well.)

\section{Frobenius SPlitting}

A Frobenius splitting $F_{*} O_{X} \rightarrow O_{X}$ is a splitting of the inclusion $O_{X} \rightarrow F_{*} O_{X}$; see $[\mathrm{MR}]$ for this concept in a geometric setting and [HR] for the commutative algebraic version. Such a splitting is easily seen to be a special type of differential operator (for details see e.g. [B]), and the well-known Frobenius splitting of toric varieties (see e.g. [HR, Theorem 5.53]) is actually induced by the globally defined differential operator $P_{0} \in \operatorname{Dist}^{(1)}(T)$ (defined in the previous section). It may be 
asked whether there are other groups with such nice elements in their algebra of distributions. But the answer is negative, as proved in the proposition below.

Let us first see that $P_{0} \in \operatorname{Dist}^{(1)}(T)$, the central idempotent corresponding to the trivial character, indeed gives such a splitting; actually we will prove this for an arbitrary toric variety $X$. This is essentially only an argument on the grading, and the normality is essential for having $P_{0}\left(O_{X}\right)=O_{X^{(1)}}$. (But see [HR].) Let the group algebra of the character group $M \cong \mathbf{Z}^{n}$ of $T$ be $k[M]:=\bigoplus_{m \in M} k e(m)$ with multiplication defined by $e\left(m_{1}\right) e\left(m_{2}\right)=e\left(m_{1}+m_{2}\right)$. The Frobenious map on $k[M]$ is determined by $F(e(m))=e(p m)$ and the splitting $P_{0}$ is given by

$$
P_{0}(e(m))= \begin{cases}e\left(m_{1}\right), & \text { if } p m_{1}=m, \\ 0, & \text { otherwise }\end{cases}
$$

Suppose that $k[S] \subset k[M]$ is the monoid algebra of a finitely generated submonoid $S \subset M$. Then the splitting $P_{0}$ clearly induces a splitting of $k[S]$ precisely when $p M \cap S=p S$. This is a property weaker than the property of $S$ to be saturated (which is the same property when $p$ is an arbitrary integer). A toric variety is constructed by gluing a set of $U_{i}=\operatorname{spec} k\left[S_{i}\right]$ for some finitely generated saturated submonoids $S_{i}$ of $M$, along open sets of the same type (cf. [O, Prop. 1.1-2]). Obviously $P_{0}$ as above defines compatible maps on each of the $k\left[S_{i}\right]$ and hence the first (well-known) part of the following proposition is clear.

Proposition 6.1. The element $P_{0} \in \operatorname{Dist}^{(1)}(T)$ induces a Frobenious splitting of all toric varieties. Torii are the only connected algebraic groups $G$ with an element $a \in \operatorname{Dist}^{1}(G)$ which induces a Frobenius splitting for $k[G]$.

Proof. An argument for the last part is almost present in the proof given in [DG, p. 509] of Nagata's theorem, mentioned earlier. Translated into terms of distributions this proof actually proceeds by proving that the assumption of linear reducibility implies the existence of a Frobenius splitting of $O_{G}$ stemming from an element which is central in the whole $\operatorname{Dist}(G)$, and that this in its turn implies that the group is a torus. But the last part of the proof is true under the slightly weaker hypothesis on the existence of splittings given in our proposition. We now give this argument, using [DG, Lemme 3.3.7] which says that it suffices to show that $G$ contains no subgroup isomorphic to ${ }_{p} \alpha_{k}$. Assume that there is such a subgroup, and that $a$ is a splitting. Then there is an inclusion of rings $\operatorname{Dist}^{(1)}\left({ }_{p} \alpha_{k}\right)=k[u] /\left(u^{p}\right) \subset \operatorname{Dist}^{(1)}(G)$ and in fact $\operatorname{Dist}^{(1)}(G)$ is a free $\operatorname{Dist}^{(1)}\left({ }_{p} \alpha_{k}\right)$-module by a Poincaré-Birkhoff-Witt theorem. Let $v_{i}, i=1, \ldots, m$, be a basis. Then $a=\sum_{i=1}^{i=m} w_{i} v_{i}, w_{i} \in \operatorname{Dist}^{(1)}\left({ }_{p} \alpha_{k}\right)$. By the property $u a=0$, it is clear that $u w_{i}=0, i=1, \ldots, m$. In particular each $w_{i} \in k u^{p-1}$ and hence $a$ is contained in the maximal ideal generated by $\operatorname{Lie}(G)$ and so $1=a(1)=0$. This is a contradiction proving the proposition.

\section{REFERENCES}

[A] E. Abe, Hopf algebras, Cambridge University Press, Cambridge, 1980. MR 83a:16010

[B] R. Bogvad, Some results on D-modules, J. Algebra 173, 638-667 (1995). MR 97a:14015

[B1] A. Borel et al., Algebraic D-modules, Academic Press (1987). MR 89g:32014

[DG] M. Demazure and P. Gabriel, Groupes algébrique, Masson/North-Holland, Paris/Amsterdam (1970). MR 46:1800

[F] W. Fulton, Introduction to toric varieties, Princeton University Press (1993). MR 94g: 14028 
[HR] M. Hochster and J. L. Roberts, The purity of the Frobenius and local cohomology, Advances in Math. 21 (1976). MR 54:5230

[J] J.-C. Jantzen, Representations of Algebraic Groups, Academic Press (1987). MR 89c:20001

[K1] T. Kaneyama, On equivariant vector bundles on an almost homogeneous variety, Nagoya Math. J. Vol. 57 (1975), 65-86. MR 51:12855

[K2] Torus-equivariant vector bundles on projective spaces, Nagoya Math. J. Vol. 111 (1988), 25-40. MR 89i:14012

[MR] V. B. Mehta, A. Ramanathan, Forbenious splitting and cohomology vanishing for Schubert varieties, Annals of Mathematics 122 (1985), p. 27-40. MR 86k:14038

[M] D. Mumford and J. Fogarty, Geometric Invariant Theory, Springer (1982). MR 86a:14006

[O] T. Oda, Convex Bodies and Algebraic Geometry, Berlin, Heidelberg, New York, Springer, 1988. MR 88m: 14038

[T] J. F. Thomsen, Frobenius direct images of line bundles on toric varieties, Preprint University of Aarhus (1996).

[Y] A. Yekutueli, An explicit construction of the Grothendieck residue complex, Astérisque 208 (1992).

Department of Mathematics, University of Stockholm, S-106 91 Stockholm, Sweden

E-mail address: rikard@matematik.su.se 